\title{
Influência do gradiente longitudinal (rio-barragem) na similaridade das comunidades de desmídias perifíticas ${ }^{1}$
}

\author{
SIRLENE APARECIDA FELISBERTO² e LILIANA RODRIGUES ${ }^{2,3}$
}

(recebido: 13 de novembro de 2003; aceito: 9 de dezembro de 2004)

\begin{abstract}
Influence of the longitudinal gradient (river-dam) in the silimarity of the communities of the periphytic desmids). The aim of this work was to evaluate periphytic desmids composition in the distinct regions to long of the axis the river-dam and the main biotic and abiotic factors that had affected the composition of this set in the Rosana (Paranapanema River) and "Salto do Vau" Reservoirs (Iguaçu River). The samples were collected in the summer and winter periods, during 2002. The substrata collected was petioles of the aquatic vegetation, in the adult stadium. In the results were registered 531 species and of these 191 are species of desmids, with 171 registered in the summer and 113 in the winter period. Of the 18 genera of desmids, Cosmarium Corda was most representative (34.2\%) in Rosana and Closterium Nitzch ex Ralfs (30.8\%) in "Salto do Vau" Reservoirs. Cluster analysis with all the showed species of desmids, sites and periods separated to both reservoirs, being that the periods were clearly evidenced only for the "Salto do Vau" Reservoir. The composition of the desmids in the three regions of Rosana varied in the two periods. Already in "Salto do Vau" the greatest richness occurred in the transition region.
\end{abstract}

Key words - aquatic macrophytes, periphytic desmids, periphyton, reservoirs

RESUMO - (Influência do gradiente longitudinal (rio-barragem) na similaridade das comunidades de desmídias perifíticas). O objetivo deste trabalho foi avaliar a composição de desmídias perifíticas nas distintas regiões ao longo do eixo rio-barragem e os principais fatores bióticos e abióticos que afetaram a composição desse conjunto nos Reservatórios de Rosana (Rio Paranapanema) e de Salto do Vau (Rio Iguaçu). As amostras foram coletadas no verão e inverno de 2002. Os substratos coletados na região litorânea foram de vegetação aquática, no estádio adulto. Do total de 531 táxons ficoperifíticos, 191 são espécies de desmídias, com 171 registradas no verão e 113 no inverno. Dos 18 gêneros de desmídias, Cosmarium Corda foi o mais representativo (34,2\%) em Rosana e Closterium Nitzch ex Ralfs (30,8\%) em Salto do Vau. A análise de agrupamento com todas as espécies de desmídias, os locais e os períodos amostrados separou ambos os reservatórios, sendo que os períodos estacionais ficaram claramente evidenciados apenas para o Reservatório de Salto do Vau. A composição das desmídias nas três regiões de Rosana variou nos dois períodos. Já em Salto do Vau, a maior riqueza ocorreu na região de transição.

Palavras-chave - desmídias perifíticas, macrófitas aquáticas, perifíton, reservatórios

\section{Introdução}

Os processos longitudinais em reservatórios, geralmente associados ao tempo de permanência da água e entrada de material alóctone, promovem o surgimento de diferentes regiões ao longo do eixo rio-barragem, denominadas de região lótica, de transição e região lêntica (Thornton 1990, Thomaz et al. 1997). Essa organização longitudinal está relacionada às características físicas e químicas, assim como aos componentes bióticos do sistema.

1. Parte da dissertação de mestrado de S.A. Felisberto no Programa de Pós-graduação em Ecologia de Ambientes Aquáticos Continentais - PEA/DBI/UEM.

2. Universidade Estadual de Maringá, Programa de Pós-graduação em Ecologia de Ambientes Aquáticos Continentais - PEA NUPÉLIA, Bloco G-90, Av. Colombo 5790, 87020-900 Maringá, PR, Brasil.

3. Autor para correspondência: 1rodrigues@ nupelia.uem.br
Assim, o conjunto das condições ambientais causa heterogeneidade espacial e temporal, promovendo uma diversidade de habitat, que é fator decisivo para a organização das comunidades e fundamental para a diversidade biológica (Tundisi 1999, Tundisi et al. 1999, Zanata \& Espíndola 2002).

Dentre os componentes bióticos destaca-se o perifíton, que representa um elo físico entre o substrato e a água circundante, onde ocorrem processos internos (autotróficos e heterotróficos) e trocas com o meio, como também com o próprio substrato, funcionando, portanto, como um microcosmo (Sládecková 1962, Wetzel 1983, Weitzel 1979).

No perifíton, as algas ganham destaque, uma vez que desempenham papel fundamental como produtoras primárias e, conseqüentemente, assumem posição chave na cadeia alimentar dos sistemas aquáticos continentais (Goldsborough \& Robinson 1996). A utilização da comunidade de algas perifíticas em monitoramento ambiental vem sendo crescente, pois, devido ao seu modo de vida séssil e grande riqueza de espécies, apresentam 
diferentes preferências e tolerâncias ambientais (Rodrigues et al. 2003).

Segundo Goldsborough \& Robinson (1996) certos fatores são determinantes na dominância de espécies em dada área incluindo a natureza do grupo (ex. epipelon, epifíton, metafíton ou fitoplâncton), a localização geográfica da área (irradiância e temperatura da água), estação do ano, profundidade da coluna d'água, hidroperíodo, tipos de macrófitas presentes e concentração de nutrientes.

Dentre as algas perifíticas, as desmídias constituem um grupo representativo em número de gêneros e espécies (Coesel 1982, 1996, Brook 1981). Geralmente esse grupo de algas ocorre em ambientes oligotróficos a eutróficos (Coesel 1982, 1996). Apesar do grande número de espécies registradas em águas ácidas (Brook 1981, Huszar 1994), muitas espécies são encontradas, com abundância considerável, em águas alcalinas (Brook 1981). Existem evidências de que algumas espécies podem sobreviver em condições de dessecação durante longos períodos (Brook \& Williamson 1988). Ainda, mudanças nas características físicas e químicas da água, como também o desaparecimento de habitats de macrófitas, podem afetar diretamente a diversidade e a composição da flórula de desmídias (Brook 1981, Coesel 1982).

No Brasil, tem-se conhecimento de apenas dois trabalhos, sob enfoque ecológico, para o grupo específico de desmídias perifíticas (Taniguchi 1998, Taniguchi et al. 2000). Para os reservatórios de Rosana (Rio Paranapanema) e Salto do Vau (Rio Iguaçu), objetos deste estudo, o conhecimento da flórula ficológica é de cunho taxonômico e restrito apenas ao primeiro reservatório citado (Bicudo et al. 1992, 1993). Esses dois últimos trabalhos foram realizados antes da construção da UHE de Rosana, ou seja, antes do represamento.

Considerando a existência de uma organização longitudinal em reservatórios, o presente estudo avaliou a composição de desmídias perifíticas nas distintas regiões ao longo do eixo rio-barragem, bem como os principais fatores bióticos e abióticos que afetaram a composição dessa comunidade nos Reservatórios de Rosana (Rio Paranapanema) e de Salto do Vau (Rio Iguaçu).

\section{Material e métodos}

Área de estudo - O Reservatório de Rosana está situado na bacia do Rio Paranapanema, o qual faz fronteira entre os Estados de São Paulo e Paraná. O Reservatório de Salto do
Vau, por sua vez, encontra-se localizado no Rio Palmital, bacia do Rio Iguaçu no estado do Paraná (figura 1).

O reservatório da Usina Hidrelétrica de Rosana situa-se no trecho do baixo Paranapanema, separando os Municípios de Diamante do Norte (Paraná) e Porto Primavera (São Paulo) entre as coordenadas $22^{\circ} 36^{\prime} \mathrm{S}$ e $52^{\circ} 50^{\prime} \mathrm{W}$ (figura 1 ). Seu substrato é composto por rocha basáltica. A usina foi inaugurada em 1987 e seu reservatório apresenta $220 \mathrm{~km}^{2} \mathrm{de}$ área, $116 \mathrm{~km}$ de extensão, profundidade de $12 \mathrm{~m}$ na região fluvial e transição, e $30 \mathrm{~m}$ na lacustre. A predominância dos usos da bacia hidrográfica, associada ao ambiente rural (agricultura, pastagem, reflorestamentos e os próprios assentamentos e propriedades rurais), é significativa, correspondendo a quase $80 \%$ de toda a área da bacia.

O reservatório da Usina Hidrelétrica de Salto do Vau (2602'12" S e 51 11'14" W) localiza-se na margem esquerda do Rio Palmital, Município de União da Vitória (Paraná) divisa com Porto União (Santa Catarina). Seu substrato é composto por rochas sedimentares. A usina foi inaugurada em 1959 com uma potência de 0,9 MW. Esse reservatório, com 8,2 km de extensão, apresenta profundidade de $0,8 \mathrm{~m}$ na região fluvial, $2 \mathrm{~m}$ na transição e $4 \mathrm{~m}$ na lacustre. A UHE de Salto do Vau foi construída numa região de vale, a qual situa-se em uma grande área de mata nativa e preservada.

Amostragem - Duas coletas foram realizadas no ano de 2002, nos períodos de verão e inverno, nas regiões lótica, de transição e lêntica dos Reservatórios de Rosana e Salto do Vau.

Os substratos coletados, sempre na região litorânea e em todos os pontos de coleta, foram pecíolos de vegetação fanerogâmica no Reservatório de Salto do Vau e Eichhornia azurea (Sw.) Kunth em Rosana. Independente da espécie, a coleta do vegetal (dois pecíolos por estação de coleta) sempre foi em estágio adulto.

O perifíton coletado, do tipo epifíton, foi removido do substrato com lâmina de barbear e jatos d'água, transferido para frascos de $150 \mathrm{~mL}$, fixado e preservado com solução de Transeau. $\mathrm{O}$ estudo taxonômico das desmídias foi obtido pela análise de aproximadamente 13 lâminas semipermanentes ou até não mais serem registradas novas espécies. Esse procedimento foi realizado em microscópio óptico com uma ocular micrometrada acoplada.

A identificação das desmídias foi baseada na literatura clássica (Förster 1982, Croasdale \& Flint 1986, 1988, Dillard 1990, 1991, Prescott et al. 1981, 1982) entre outras. O enquadramento taxonômico adotado foi o proposto por Förster (1982). A organização das espécies dentro de cada família foi estabelecida em ordem alfabética.

Os dados abióticos foram coletados simultaneamente aos dados bióticos e fornecidos pelo Laboratório de Limnologia Básica, do Núcleo de Pesquisas em Limnologia, Ictiologia e Aquicultura - Nupélia, da Universidade Estadual de Maringá. Os parâmetros físicos e químicos utilizados foram: transparência (Disco de Secchi), temperatura da água e oxigênio dissolvido (Oxímetro portátil marca YSI modelo 55), 


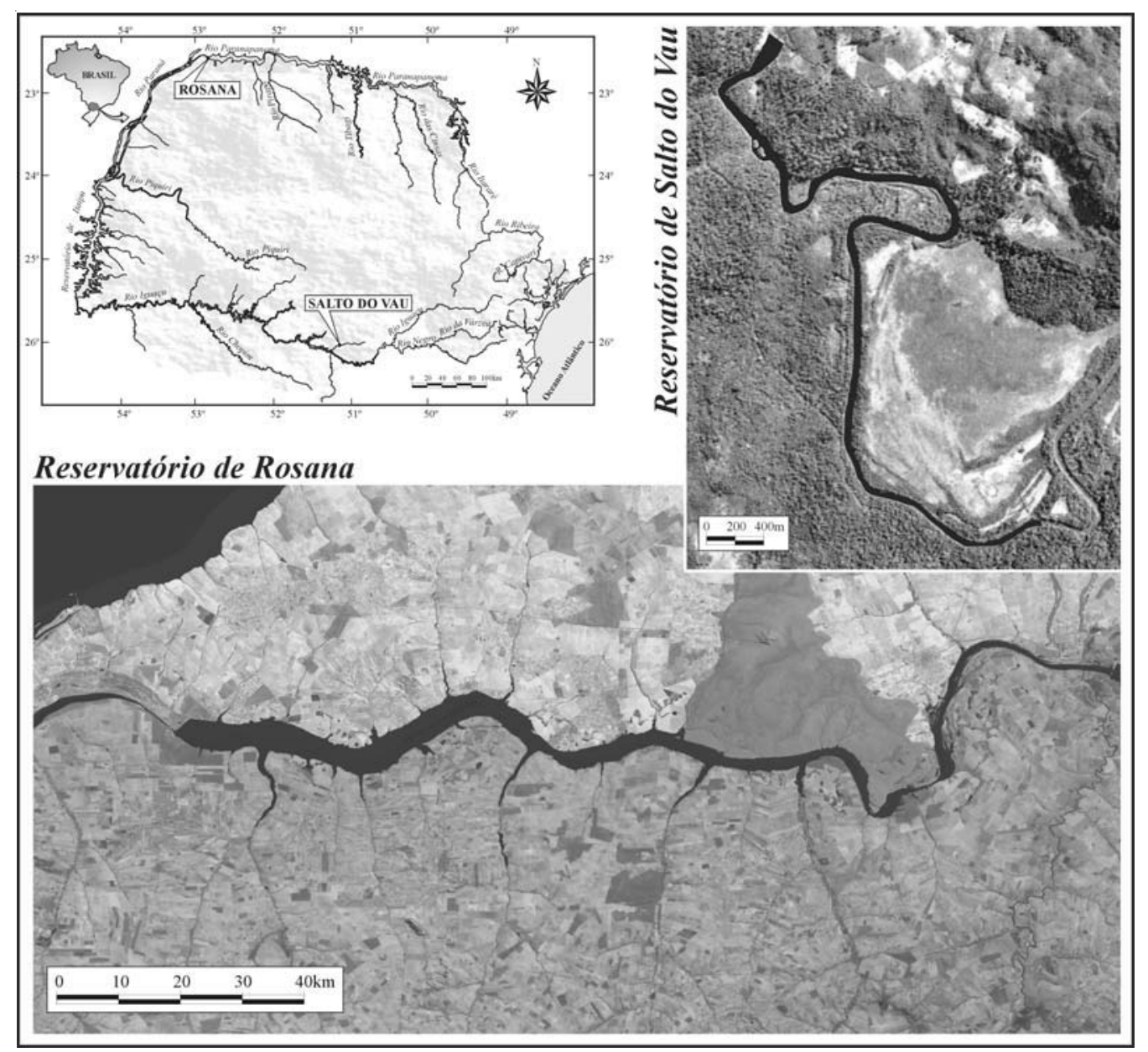

Figura 1. Localização dos Reservatórios Rosana (Bacia do Rio Paranapanema) e Salto do Vau (Bacia do Rio Iguaçu), no Sudeste do Brasil.

Figure 1. Localization of the Rosana (Paranapanema River Basin) and Salto do Vau (Iguaçu River Basin) Reservoirs, in the southeast of the Brazil.

condutividade elétrica (Condutivímetro portátil Digimed modelo DM2), $\mathrm{pH}$ (pHmetro portátil Digimed modelo DM2), turbidez (Turbidímetro portátil modelo Lamotte). Os dados referentes às macrófitas aquáticas foram cedidos pelo Laboratório de Macrófitas Aquáticas desse mesmo Núcleo. Análise dos dados - A similaridade das desmídias entre os reservatórios, os períodos estacionais e as regiões ao longo da distribuição longitudinal (rio-barragem) foi medida pela análise de agrupamento. Nessa análise foi utilizado o critério de presença e ausência através da distância pelo índice de Jaccard de acordo com o programa NTSYS, versão 1.5 (Rohlf 1989) e média não-ponderada (UPGMA). O número de táxons de macrófitas aquáticas e os dados abióticos foram correlacionados com o de táxons de desmídias, utilizando o coeficiente de correlação de Pearson, a fim de analisar a influência dessas variáveis sobre a composição de desmídias (nível de significância $\mathrm{p}<0,05 \%$ ). Para esse teste utilizou-se o programa Statistica versão 5.0 (StatSoft Inc. 1996).

\section{Resultados}

Caracterização limnológica - Dentre os valores referentes aos parâmetros físicos e químicos da água, a temperatura e a condutividade elétrica apresentaram maior variação entre os reservatórios e entre as duas estações do ano. Assim, no Reservatório de Salto do Vau foram registradas baixas temperaturas, principalmente no inverno, enquanto no Reservatório de Rosana não ocorreram oscilações acentuadas entre as estações (tabela 1).

Para a condutividade elétrica, baixos valores (a metade dos valores registrados para o Reservatório de Rosana) foram observados nos dois períodos para o Reservatório de Salto do Vau. Os valores de turbidez e oxigênio dissolvido foram mais elevados em Salto do Vau, principalmente no inverno, enquanto a 
Tabela 1. Variáveis limnológicas medidas na sub-superfície das diferentes regiões dos dois reservatórios, durante o ano de 2002. (Cond. $=$ Condutividade elétrica, OD. $=$ Oxigênio Dissolvido, Temp. $=$ Temperatura da água, $\mathrm{t}=$ Secchi total (transparência da água)). (Fonte: Laboratório de Limnologia/Nupélia).

Table 1. Limnologic variable measures in the subsurface of the different regions of the two reservoirs, during the year of 2002. $($ Cond.$=$ electrical conductivity, DO. $=$ dissolved oxygen, Temp. $=$ Water Temperature, $\mathrm{t}=$ total secchi $($ water transparency) $)$. (Source: Laboratory of Limnology/Nupélia).

\begin{tabular}{lllcccc}
\hline Reservatórios/regiões & $\begin{array}{c}\text { Secchi } \\
(\mathrm{m})\end{array}$ & $\begin{array}{c}\text { Cond. } \\
(\mu \mathrm{S} \mathrm{cm})\end{array}$ & $\mathrm{pH}$ & $\begin{array}{c}\text { Turbidez } \\
(\mathrm{NTU})\end{array}$ & $\begin{array}{c}\text { OD. } \\
\left(\mathrm{mg} \mathrm{L}^{-1}\right)\end{array}$ & $\begin{array}{c}\text { Temp. } \\
\left({ }^{\circ} \mathrm{C}\right)\end{array}$ \\
\hline Verão & & & & & & \\
$\quad$ Salto do Vau Fluvial & 0,8 & 23,2 & 6,8 & 4,7 & 8,0 & 21,8 \\
$\quad$ Salto do Vau Transição & 1,0 & 23,3 & 7,4 & 3,6 & 7,5 & 23,2 \\
$\quad$ Salto do Vau Lacustre & 1,1 & 23,7 & 7,4 & 3,5 & 6,9 & 23,7 \\
Rosana Fluvial & 1,9 & 69,1 & 7,4 & 1,7 & 6,6 & 26,8 \\
Rosana Transição & 2,1 & 63,4 & 7,5 & 1,4 & 7,3 & 27,1 \\
Rosana Lacustre & 2,6 & 62,8 & 7,5 & 1,3 & 7,5 & 27,7 \\
Inverno & & & & & & \\
$\quad$ Salto do Vau Fluvial & $\mathrm{t}$ & 19,6 & 6,8 & 2,3 & 9,5 & 13,3 \\
Salto do Vau Transição & 1,9 & 19,7 & 6,3 & 2,1 & 9,0 & 13,0 \\
Salto do Vau Lacustre & 2,0 & 26,3 & 6,1 & 2,2 & 9,6 & 13,6 \\
Rosana Fluvial & 2,4 & 57,2 & 7,2 & 1,5 & 8,2 & 21,7 \\
Rosana Transição & 2,7 & 50,3 & 6,8 & 1,2 & 8,3 & 20,9 \\
Rosana Lacustre & 2,2 & 60,5 & 7,4 & 1,5 & 9,1 & 24,5 \\
\hline
\end{tabular}

transparência da água apresentou baixos valores nesse período (tabela 1).

No Reservatório de Rosana foram identificados 24 táxons de macrófitas aquáticas, sendo constatados no período de verão oito na região fluvial, 11 na de transição e 10 na lacustre. No inverno, foram registrados 14 táxons na região fluvial, 11 na região de transição e 15 na lacustre. Por sua vez, no Reservatório de Salto do Vau foram registrados apenas sete táxons de macrófitas, assim distribuídos: três na fluvial, quatro na transição e sete na lacustre no verão; três na fluvial, um na transição e dois na lacustre no inverno (S.M. Thomaz, dados não publicados).

Composição das Desmídias na comunidade epifítica - A análise taxonômica das amostras das três regiões dos reservatórios de Rosana e Salto do Vau permitiu o reconhecimento de 531 táxons, considerando os dois períodos amostrados. Desse total, 403 ocorreram no Reservatório de Rosana e 295 no Reservatório de Salto do Vau (S.A. Felisberto, dados não publicados).

Do total de algas perifíticas, a contribuição das desmídias foi de 38\% (155 táxons de desmídias e 248 de outros grupos) no Reservatório de Rosana (Rio Paranapanema) (figura 2A) e 27\% (81 desmídias e 214 outros grupos) no Reservatório de Salto do Vau (Rio Iguaçu) (figura 2B). Dessa forma, a riqueza das desmídias foi maior no primeiro reservatório.

A composição das desmídias nas três regiões do Reservatório de Rosana variou nos dois períodos, com uma diferença mais acentuada ao longo do eixo longitudinal no período de inverno. Já em Salto do Vau, a maior riqueza ocorreu na região de transição independente do período estacional (tabela 2).

Considerando os dois reservatórios, foram registrados 18 gêneros: Actinotaenium (Nägeli) Teiling, Closterium Nitzsch ex Ralfs, Cosmarium Corda, Cosmocladium Brébisson, Cylindrocystis Meneghini, Desmidium C. Agardh, Euastrum Ehrenberg, Gonatozygon Bary, Hyalotheca Ehrenberg, Micrasterias Ralfs, Netrium (Nägeli) Itzigsohn, Onychonema Wallich, Penium Brébisson ex Ralfs, Pleurotaenium, Sphaerozosma, Spondylosium, Staurastrum Meyen e Staurodesmus Teilling. Independente da região, no Reservatório de Rosana, Cosmarium e Staurastrum foram os mais representativos em espécies, com $34,2 \%$ e $20 \%$, respectivamente, enquanto no Reservatório de Salto do Vau o gênero Closterium apresentou expressiva riqueza específica, seguido de Cosmarium (tabela 3, figura 3).

Do total de táxons de desmídias inventariados, 44 ocorreram em ambos os reservatórios analisados, independente da região e do período estacional 

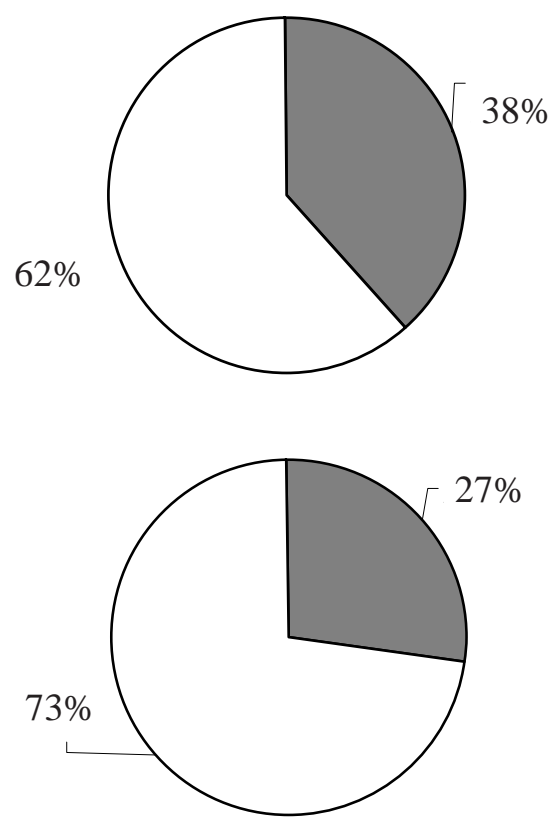

B

Figura 2. Riqueza específica (\%) das desmídias em relação à comunidade de algas perifíticas encontrada nos reservatórios de Rosana (A) e Salto do Vau (B), durante o ano de 2002. = desmídias; $\square=$ outros grupos.

Figure 2. Specific richness (\%) of the desmids in relation to the community of periphytic algae found in Rosana (A) and Salto do Vau (B) reservoirs, during the year of 2002. $\square$ = desmids; $\square=$ groups other.

(tabela 3). A maior frequiência de ocorrência foi de Cosmarium abbreviatum Raciborski var. minus (West $\&$ West) Krieger \& Gerloff (tabela 3).

Constatou-se a presença de 34 táxons de ocorrência exclusiva para o Reservatório de Salto do Vau (tabela 3). O gênero Closterium apresentou 16 táxons específicos e infraespecíficos. Duas espécies, Euastrum denticulatum (Kirchner) Gay e Hyalotheca dissiliens (Smith) Brébisson ex Ralfs apresentaram 100\% de ocorrência, ou seja, ocorreram em ambos os períodos e em todos os pontos de coleta. Closterium ehrenbergii Meneghini ex Ralfs, C. incurvum Brébisson, C. moniliferum (Bory) Ehrenberg ex Ralfs, Cosmarium abbreviatum var. minus e Pleurotaenium ehrenbergii (Brébisson) De Bary ocorreram em 83,3\% das amostras (tabela 3).

No Reservatório de Rosana, por sua vez, foram constatados 110 táxons de ocorrência exclusiva (tabela 3). O gênero com maior número de táxons foi Cosmarium. As espécies Cosmarium abbreviatum var. minus, C. granatum Brébisson ex Ralfs,
Tabela 2. Riqueza específica de desmídias encontrada nos reservatórios de Rosana e Salto do Vau, por período estacional (verão e inverno).

Table 2. Specific richness of desmids found on the two studied reservoirs, in Summer and carried out in Winter period.

\begin{tabular}{lcc}
\hline & Rosana & Salto do Vau \\
\hline Verão & & \\
$\quad$ Região fluvial & 54 & 17 \\
Região de transição & 56 & 52 \\
$\quad$ Região lacustre & 106 & 45 \\
Inverno & & \\
$\quad$ Região fluvial & 22 & 20 \\
Região de transição & 40 & 29 \\
Região lacustre & 76 & 13 \\
\hline
\end{tabular}

C. pseudoconnatum Nordstedt, C. punctulatum Brébisson, $C$. quadrum Lundell var. minus Nordstedt e Staurastrum cf. dilatatum (Ehrenberg) Ralfs ocorreram ao longo do eixo longitudinal do reservatório e em ambos os períodos estacionais, apresentando assim 100\% de freqüência de ocorrência (tabela 3 ).

Índice de similaridade e análise de agrupamento de espécies - Dois grandes grupos foram formados inicialmente, discriminando o conjunto de desmídias perifíticas de ambos os reservatórios. Para o reservatório da UHE de Rosana, a formação de grupos se deu em função das regiões ao longo do eixo longitudinal, seguida do período estacional. Para o reservatório da UHE de Salto do Vau observou-se o contrário: as desmídias foram caracterizadas principalmente pela estação do ano e, em seguida, pelas regiões, ao longo do eixo longitudinal do reservatório (figura 4).

Correlação entre Desmídias perifíticas e as variáveis limnológicas - Dentre as variáveis analisadas nos reservatórios, a composição específica das desmídias foi correlacionada positivamente com a temperatura da água $(\mathrm{r}=0,73), \mathrm{pH}(\mathrm{r}=0,71)$ e condutividade elétrica $(\mathrm{r}=0,61)$; houve também correlação com a riqueza de espécies de macrófitas aquáticas $(r=0,59)$.

De forma geral, o Reservatório de Salto do Vau apresentou menor quantidade de macrófitas em toda sua extensão, implicando também em um menor número de espécies de desmídias perifíticas. Além disso, independente da região ao longo do eixo longitudinal, o período de menores temperaturas (inverno) caracterizou menor número de espécies de ambas as comunidades (figura 5). No Reservatório de Rosana não ocorreu uma distinção entre os períodos estacionais. Na região lêntica, 
Tabela 3. Táxons de desmídias (Conjugatophyceae) encontrados nos dois reservatórios e suas respectivas ocorrências nas diferentes regiões: Fluvial (F), Transição (T), Lacustre (L).

Table 3. Taxa of desmids (Conjugatophyceae) found in the two studied reservoirs and their respective occurrence on the various regions: Fluvial (F), Transition (T), Lacustrine (L).

\begin{tabular}{|c|c|c|c|c|c|c|c|c|c|c|c|c|}
\hline \multirow{3}{*}{$\begin{array}{l}\text { Reservatórios } \\
\text { Períodos estacionais } \\
\text { Família/Táxons }\end{array}$} & \multicolumn{6}{|c|}{ Rosana } & \multicolumn{6}{|c|}{ Salto do Vau } \\
\hline & \multicolumn{3}{|c|}{ Verão } & \multicolumn{3}{|c|}{ Inverno } & \multicolumn{3}{|c|}{ Verão } & \multicolumn{3}{|c|}{ Inverno } \\
\hline & $\mathrm{F}$ & $\mathrm{T}$ & $\mathrm{L}$ & $\mathrm{F}$ & $\mathrm{T}$ & $\mathrm{L}$ & $\mathrm{F}$ & $\mathrm{T}$ & $\mathrm{L}$ & $\mathrm{F}$ & $\mathrm{T}$ & $\mathrm{L}$ \\
\hline \multicolumn{13}{|l|}{ MESOTAENIACEAE } \\
\hline Cylindrocystis brebissonii (Menegh. ex Ralfs) de Bary & & & & & & & & & $\mathrm{x}$ & & $\mathrm{x}$ & \\
\hline Netrium digitus (Ehrenb.) Itzigs. \& Rothe & $\mathrm{x}$ & & $\mathrm{x}$ & & & & & & $\mathrm{x}$ & & & \\
\hline \multicolumn{13}{|l|}{ GONATOZYGACEAE } \\
\hline Gonatozygon aculeatum Hast. & & & $\mathrm{x}$ & & & & & & & & & \\
\hline G. brebissonii de Bary & & & $\mathrm{x}$ & & & & & & & & & \\
\hline G. monotaenium de Bary & $\mathrm{x}$ & $\mathrm{x}$ & $\mathrm{x}$ & & & $\mathrm{x}$ & & $\mathrm{x}$ & $\mathrm{x}$ & $\mathrm{x}$ & $\mathrm{x}$ & \\
\hline G. pilosum Wolle & $\mathrm{x}$ & $\mathrm{x}$ & & & & & & & & & & \\
\hline \multicolumn{13}{|l|}{ PENIACEAE } \\
\hline Penium margaritaceum (Ehrenb.) ex Bréb. & $\mathrm{x}$ & & $\mathrm{x}$ & $\mathrm{x}$ & & & & & & & & \\
\hline \multicolumn{13}{|l|}{ CLOSTERIACEAE } \\
\hline $\begin{array}{l}\text { Closterium acutum Ehrenb. var. variabile (Lemmerm.) } \\
\text { W. Krieg. }\end{array}$ & & & & & & & $\mathrm{x}$ & & & & & \\
\hline C. archerianum Cleve & & & & & & & & & $\mathrm{x}$ & & & \\
\hline C. calosporum Wittr. & $\mathrm{x}$ & $\mathrm{x}$ & & & & & & & & & & \\
\hline C. closterioides (Ralfs) A. Louis \& Peet. & & & & & & & & & $\mathrm{x}$ & & & \\
\hline C. cornu Ehrenb. ex Ralfs & & & & & & $\mathrm{x}$ & & $\mathrm{x}$ & & & & \\
\hline C. costatum Corda ex Ralfs & & & & & & & & & $\mathrm{x}$ & & & \\
\hline C. cynthia de Not. & & & & & & & & $\mathrm{x}$ & $\mathrm{x}$ & & $\mathrm{x}$ & $\mathrm{x}$ \\
\hline C. dianae Ehrenb. ex Ralfs var. dianae & & & $\mathrm{x}$ & & & & & $\mathrm{x}$ & & & & $\mathrm{x}$ \\
\hline C. dianae Ehrenb. ex Ralfs var. compressum Klebs & & & & & & & & $\mathrm{x}$ & & & & \\
\hline C. dianae Ehrenb. ex Ralfs var. minus Hieron. & & & $\mathrm{x}$ & & & & & & & & $\mathrm{x}$ & \\
\hline C. ehrenbergii Menegh. ex Ralfs & & & & & & & $\mathrm{x}$ & & $\mathrm{x}$ & $\mathrm{x}$ & $\mathrm{x}$ & $\mathrm{x}$ \\
\hline C. exiguum West \& G.S.West & & & $\mathrm{x}$ & & & & & & & & & \\
\hline C. incurvum Bréb. & $\mathrm{x}$ & $\mathrm{x}$ & $\mathrm{x}$ & & $\mathrm{x}$ & $\mathrm{x}$ & $\mathrm{x}$ & $\mathrm{x}$ & $\mathrm{x}$ & $\mathrm{x}$ & $\mathrm{x}$ & \\
\hline C. cf. intermedium Ralfs & & & & & & & & & $\mathrm{x}$ & $\mathrm{x}$ & $\mathrm{x}$ & $\mathrm{x}$ \\
\hline C.jenneri Ralfs & & & & & & & & $\mathrm{x}$ & & & & \\
\hline C. laterale Nordst. var. laterale & & & & & & & $\mathrm{x}$ & & & & $\mathrm{x}$ & \\
\hline C. leibleinii Kütz. ex Ralfs & $\mathrm{x}$ & & & & & & & & & & & \\
\hline C. lunula (Müll.) Nitzsch ex Ralfs var. intermedium Gutw. & & & & & & & $\mathrm{x}$ & & & & & \\
\hline C. moniliferum (Bory) Ehrenb. ex Ralfs & $\mathrm{x}$ & & & $\mathrm{x}$ & & & $\mathrm{x}$ & $\mathrm{x}$ & $\mathrm{x}$ & $\mathrm{x}$ & $\mathrm{x}$ & \\
\hline C. navicula (Bréb.) Lütkem. & & & & & & & & $\mathrm{x}$ & $\mathrm{x}$ & $\mathrm{x}$ & $\mathrm{x}$ & \\
\hline C. nematodes Josh. var. proboscideum Turn. & & & & & & & $\mathrm{x}$ & $\mathrm{x}$ & $\mathrm{x}$ & & $\mathrm{x}$ & \\
\hline C. pusillum Hantzsch & & & & & & & & $\mathrm{x}$ & & & & \\
\hline C. ralfsii Bréb. var. hybridum Rabenh. & & & & & & & $\mathrm{x}$ & & & & & \\
\hline C. setaceum Ehrenb. ex Ralfs & & & & & & & & $\mathrm{x}$ & $\mathrm{x}$ & & & \\
\hline C. striolatum Ehrenb. ex Ralfs & & & & & & & & $\mathrm{x}$ & $\mathrm{x}$ & & & \\
\hline C. tortum B.M. Griffiths & & & & $\mathrm{x}$ & & $\mathrm{x}$ & & & & & & \\
\hline C. toxon W. West & & & & & & & & $\mathrm{x}$ & $\mathrm{x}$ & $\mathrm{x}$ & $\mathrm{x}$ & \\
\hline C. tumidum Johns. var. nylandicum Grönbl. & & $\mathrm{x}$ & $\mathrm{x}$ & & & $\mathrm{x}$ & & $\mathrm{x}$ & & & & \\
\hline C. venus Kütz. ex Ralfs & $\mathrm{x}$ & & $\mathrm{x}$ & & & & & & & & & \\
\hline C. venus Kütz. ex Ralfs var. westii W. Krieg. & & & $\mathrm{x}$ & & & & & & & & & \\
\hline Closterium sp. & & $\mathrm{x}$ & & & & & & & & & & \\
\hline
\end{tabular}


continuação

\begin{tabular}{|c|c|c|c|c|c|c|c|c|c|c|c|c|}
\hline \multirow{3}{*}{$\begin{array}{l}\text { Reservatórios } \\
\text { Períodos estacionais } \\
\text { Família/Táxons }\end{array}$} & \multicolumn{6}{|c|}{ Rosana } & \multicolumn{6}{|c|}{ Salto do Vau } \\
\hline & \multicolumn{3}{|c|}{ Verão } & \multicolumn{3}{|c|}{ Inverno } & \multicolumn{3}{|c|}{ Verão } & \multicolumn{3}{|c|}{ Inverno } \\
\hline & $\mathrm{F}$ & $\mathrm{T}$ & $\mathrm{L}$ & $\mathrm{F}$ & $\mathrm{T}$ & $\mathrm{L}$ & $\mathrm{F}$ & $\mathrm{T}$ & $\mathrm{L}$ & $\mathrm{F}$ & $\mathrm{T}$ & $\mathrm{L}$ \\
\hline \multicolumn{13}{|l|}{ DESMIDIACEAE } \\
\hline Actinotaenium diplosporum (Lund.) Teil. & & & $\mathrm{x}$ & & & & & & & & & \\
\hline A. globosum (Bulnh.) Teil. & $\mathrm{X}$ & & $\mathrm{x}$ & & & & & $\mathrm{x}$ & $\mathrm{x}$ & & & \\
\hline Actinotaenium sp. & & & & & & & & & $\mathrm{x}$ & & & \\
\hline $\begin{array}{l}\text { Cosmarium abbreviatum Racib. var. minus (West \& G.S. West) } \\
\text { W. Krieg. \& Gerl. }\end{array}$ & $\mathrm{x}$ & $\mathrm{x}$ & $\mathrm{x}$ & $\mathrm{x}$ & $\mathrm{x}$ & $\mathrm{x}$ & $\mathrm{x}$ & $\mathrm{X}$ & $\mathrm{x}$ & $\mathrm{X}$ & $\mathrm{X}$ & \\
\hline C. cf. adoxum West \& G.S. West & & & $\mathrm{x}$ & & & & & & & & & \\
\hline C. anisochondrum Nordst. var. tetrachondrum Scott \& Grönbl. & $\mathrm{X}$ & $\mathrm{X}$ & $\mathrm{x}$ & & & $\mathrm{X}$ & & & & & & \\
\hline C. baileyi Wolle & $\mathrm{X}$ & $\mathrm{X}$ & & $\mathrm{x}$ & & & & & & & & \\
\hline C. bioculatum (Bréb.) ex Ralfs * & $\mathrm{X}$ & & & & & & & $\mathrm{x}$ & & & & \\
\hline C. blyttii Wille * & $\mathrm{X}$ & $\mathrm{X}$ & $\mathrm{x}$ & & & & & & & & & \\
\hline C. comissurale (Bréb.) Ralfs var. crassum Nordst. & & & & & & & & $\mathrm{x}$ & & & & \\
\hline C. cf. connatum (Bréb.) Ralfs & & & & & & $\mathrm{x}$ & & & & & & \\
\hline C. excavatum Nordst. & & & $\mathrm{x}$ & & & & & & & & & \\
\hline C. exiguum Arch. & & & $\mathrm{x}$ & & $\mathrm{x}$ & $\mathrm{x}$ & & $\mathrm{X}$ & & & & \\
\hline C. galeritum Nordst. var. subtumidum Borge & $\mathrm{X}$ & $\mathrm{X}$ & $\mathrm{x}$ & & $\mathrm{x}$ & $\mathrm{x}$ & & & & & & \\
\hline C. granatum Bréb. ex Ralfs * & $\mathrm{x}$ & $\mathrm{x}$ & $\mathrm{x}$ & $\mathrm{x}$ & $\mathrm{x}$ & $\mathrm{x}$ & & & & & & \\
\hline C. impressulum Elfv. var. impressulum* & & $\mathrm{x}$ & $\mathrm{x}$ & $\mathrm{X}$ & $\mathrm{x}$ & $\mathrm{x}$ & & & & & & \\
\hline C. impressulum Elfv. var. alpicolum Schmidle & $\mathrm{X}$ & $\mathrm{X}$ & & & $\mathrm{x}$ & $\mathrm{x}$ & & & & & & \\
\hline C. laeve Rabenh. var. laeve* & $\mathrm{x}$ & & $\mathrm{x}$ & $\mathrm{X}$ & $\mathrm{x}$ & $\mathrm{x}$ & & $\mathrm{x}$ & $\mathrm{x}$ & $\mathrm{x}$ & & \\
\hline C. laeve Rabenh. var. westii W. Krieg. \& Gerl. & $\mathrm{x}$ & & & & & & & & & & & \\
\hline C. lagoense (Nordst.) Nordst. var. amoebum Först. \& Eck. & & & $\mathrm{x}$ & & & & & & & & & \\
\hline C. cf. logiense Biss. & & & & & & $\mathrm{x}$ & & & & & & \\
\hline C. mamilliferum Nordst. & & $\mathrm{x}$ & $\mathrm{x}$ & & & & & & & & & \\
\hline $\begin{array}{l}\text { C. margaritatum (Lund.) Roy \& Biss. var. margaritatum } \\
\text { f. minor (Boldt) West \& G.S. West }\end{array}$ & & & $\mathrm{x}$ & & & & & & & & & \\
\hline C. moerlianum Lütk. var. brasiliense Borge & $\mathrm{X}$ & $\mathrm{x}$ & $\mathrm{x}$ & & $\mathrm{x}$ & $\mathrm{x}$ & & & & & & \\
\hline C. naegelianum Bréb. * & $\mathrm{X}$ & & & $\mathrm{X}$ & & & & & & & & \\
\hline $\begin{array}{l}\text { C. norimbergense Reinsch var. depressum (West \& } \\
\text { G.S. West) W. Krieg. \& Gerl. }\end{array}$ & & $\mathrm{X}$ & $\mathrm{x}$ & & $\mathrm{x}$ & $\mathrm{x}$ & & & & & & \\
\hline C. ovale Ralfs & & & & & & $\mathrm{x}$ & & & & & & \\
\hline C. pachydermum Lund. * & & $\mathrm{x}$ & $\mathrm{x}$ & & & & & & & & & \\
\hline C. pachydermum Lund. f. parvum Croasd. & $\mathrm{X}$ & & & & & & & & & & & \\
\hline C. porrectum Nordst. & & $\mathrm{x}$ & $\mathrm{x}$ & & $\mathrm{x}$ & & & & & & & \\
\hline C. portianum Arch. * & & $\mathrm{X}$ & $\mathrm{x}$ & & $\mathrm{x}$ & $\mathrm{x}$ & & & & & & \\
\hline C. protractum (Näg.) de Bary & & & & & $\mathrm{x}$ & & & & & & & \\
\hline C. pseudoconnatum Nordst. * & $\mathrm{x}$ & $\mathrm{x}$ & $\mathrm{x}$ & $\mathrm{x}$ & $\mathrm{x}$ & $\mathrm{x}$ & $\mathrm{x}$ & $\mathrm{x}$ & $\mathrm{x}$ & & & \\
\hline C. pseudoexiguum Racib. & & & $\mathrm{x}$ & & $\mathrm{x}$ & & & & & & & \\
\hline C. pseudoprotuberans Kirchn. var. notatum Skuja & & & $\mathrm{x}$ & & & $\mathrm{x}$ & & & & & & \\
\hline C. punctulatum Bréb. * & $\mathrm{x}$ & $\mathrm{x}$ & $\mathrm{x}$ & $\mathrm{x}$ & $\mathrm{x}$ & $\mathrm{x}$ & & $\mathrm{x}$ & $\mathrm{X}$ & & & $\mathrm{x}$ \\
\hline C. cf. pyramidatum Bréb. (Ralfs) & & & & & & & $\mathrm{x}$ & & $\mathrm{X}$ & & & \\
\hline C. quadrum Lund. var. minus Nordst. * & $\mathrm{x}$ & $\mathrm{x}$ & $\mathrm{x}$ & $\mathrm{x}$ & $\mathrm{x}$ & $\mathrm{x}$ & & & & & & \\
\hline C. quadrum Lund. var. sublatum (Nordst.) West \& G.S. West & & $\mathrm{X}$ & $\mathrm{x}$ & & $\mathrm{x}$ & $\mathrm{x}$ & & & & & & \\
\hline C. quinarium Lund. & & & $\mathrm{x}$ & & & & & & & & & \\
\hline C. ralfsii Bréb. var. montanum Racib. & & $\mathrm{X}$ & & & & & & & & & & \\
\hline $\begin{array}{l}\text { C. rectangulare Grun. var. hexagonum (Elfv.) West \& } \\
\text { G.S. West }\end{array}$ & $\mathrm{x}$ & & & $\mathrm{x}$ & $\mathrm{x}$ & & & & & & & \\
\hline C. regnellii Wille var. regnellii $*$ & & & $\mathrm{x}$ & & $\mathrm{x}$ & & & $\mathrm{x}$ & & $\mathrm{X}$ & & \\
\hline C. regnellii Wille var. pseudoregnellii (Messik.) & & & $\mathrm{x}$ & $\mathrm{x}$ & & $\mathrm{x}$ & & & & & & \\
\hline
\end{tabular}


continuação

\begin{tabular}{|c|c|c|c|c|c|c|c|c|c|c|c|c|}
\hline \multirow{3}{*}{$\begin{array}{l}\text { Reservatórios } \\
\text { Períodos estacionais } \\
\text { Família/Táxons }\end{array}$} & \multicolumn{6}{|c|}{ Rosana } & \multicolumn{6}{|c|}{ Salto do Vau } \\
\hline & \multicolumn{3}{|c|}{ Verão } & \multicolumn{3}{|c|}{ Inverno } & \multicolumn{3}{|c|}{ Verão } & \multicolumn{3}{|c|}{ Inverno } \\
\hline & $\mathrm{F}$ & $\mathrm{T}$ & $\mathrm{L}$ & $\mathrm{F}$ & $\mathrm{T}$ & $\mathrm{L}$ & $\mathrm{F}$ & $\mathrm{T}$ & $\mathrm{L}$ & $\mathrm{F}$ & $\mathrm{T}$ & $\mathrm{L}$ \\
\hline C. regnesii Reinsch * & $\mathrm{x}$ & $\mathrm{x}$ & $\mathrm{X}$ & & & $\mathrm{x}$ & & $\mathrm{x}$ & & & & \\
\hline C. reniforme (Ralfs) Arch. var. reniforme* & $\mathrm{x}$ & $\mathrm{x}$ & & & $\mathrm{x}$ & $\mathrm{x}$ & & $\mathrm{x}$ & & $\mathrm{x}$ & $\mathrm{x}$ & $\mathrm{x}$ \\
\hline C. reniforme (Ralfs) Arch. var. compressum Nordst. & & & & & $\mathrm{x}$ & & & & & & & \\
\hline C. reniforme (Ralfs) Arch. ** & & $\mathrm{x}$ & & & & & & & & & & \\
\hline C. sexnotatum Gütw. var. tristiatum (Lütkem.) Schmidle & & $\mathrm{x}$ & & & & & & & & & & \\
\hline C. cf. speciosum Lund. & & & $\mathrm{X}$ & & & & & & & & & \\
\hline C. subspeciosum Nordst. var. subspeciosum * & & & $\mathrm{x}$ & & & & & $\mathrm{x}$ & $\mathrm{x}$ & $\mathrm{X}$ & & \\
\hline C. subspeciosum Nordst. var. validius Nordst. * & & & & & & & & $\mathrm{x}$ & $\mathrm{x}$ & & $\mathrm{x}$ & \\
\hline C. subtumidum Nordst. * & $\mathrm{X}$ & & $\mathrm{x}$ & & $\mathrm{x}$ & & & $\mathrm{x}$ & & & & \\
\hline C. trilobulatum Reinsch & $\mathrm{x}$ & $\mathrm{x}$ & $\mathrm{x}$ & & $\mathrm{x}$ & $\mathrm{x}$ & & & & & & \\
\hline C. vexatum $\mathrm{W}$. West $*$ & $\mathrm{x}$ & & & $\mathrm{x}$ & & & & $\mathrm{x}$ & $\mathrm{x}$ & & & $\mathrm{X}$ \\
\hline Cosmarium sp. & & & $\mathrm{x}$ & & & & & & & & & \\
\hline Cosmarium sp.1 & & & $\mathrm{X}$ & & & $\mathrm{X}$ & & & & & & \\
\hline Cosmarium sp.2 & $\mathrm{x}$ & & & & & & & & $\mathrm{x}$ & & & \\
\hline Cosmarium sp.3 & & & & & & & & $\mathrm{x}$ & & & & \\
\hline Cosmarium sp.4 & & & & & $\mathrm{x}$ & $\mathrm{x}$ & & & & & & \\
\hline Desmidium cf. aptogonum Bréb. & & & $\mathrm{x}$ & & & $\mathrm{X}$ & & & & & & \\
\hline D. baileyi (Ralfs) Nordst. & & & $\mathrm{x}$ & & & $\mathrm{x}$ & & & & & & \\
\hline D. pseudostreptonema West \& G.S. West & & $\mathrm{x}$ & $\mathrm{x}$ & & & $\mathrm{X}$ & & & & & & \\
\hline Euastrum abruptum Nordst. var. abruptum & $\mathrm{x}$ & & $\mathrm{x}$ & $\mathrm{x}$ & $\mathrm{x}$ & $\mathrm{x}$ & & & & & & \\
\hline E. abruptum Nordst. var. subglaziowii (Borge) W. Krieg. & & $\mathrm{x}$ & $\mathrm{x}$ & & & $\mathrm{x}$ & & & & & & \\
\hline E. denticulatum (Kirchn.) Gay & $\mathrm{x}$ & & & & & $\mathrm{x}$ & $\mathrm{x}$ & $\mathrm{x}$ & $\mathrm{x}$ & $\mathrm{x}$ & $\mathrm{x}$ & $\mathrm{X}$ \\
\hline E. gayanum de Toni var. angulatum W. Krieg. & $\mathrm{x}$ & & $\mathrm{x}$ & & $\mathrm{x}$ & $\mathrm{x}$ & & & & & & \\
\hline E. hypochondrum Nordst. & & $\mathrm{x}$ & & & & & & & & & & \\
\hline E. insulare (Wittr.) Roy & & & & & & & & $\mathrm{x}$ & & & & \\
\hline E. monocylum (Nordst.) Racib. var. borgei Grönbl. & & & & & & & & $\mathrm{x}$ & $\mathrm{x}$ & & & \\
\hline E. subintegrum Nordst. var. brasiliense Grönbl. & $\mathrm{x}$ & & & & & & & & & & & \\
\hline Hyalotheca dissiliens (Smith) Bréb. ex Ralfs & & $\mathrm{x}$ & & & & $\mathrm{x}$ & $\mathrm{x}$ & $\mathrm{x}$ & $\mathrm{x}$ & $\mathrm{x}$ & $\mathrm{x}$ & $\mathrm{X}$ \\
\hline Micrasterias abrupta West \& G.S. West & $\mathrm{x}$ & & & & & & & & & & & \\
\hline M. borgei W. Krieg. & & $\mathrm{x}$ & & & & & & & & & & \\
\hline M. excavata (Nordst.) C. Bic. \& Sorm. & & & & & & & & & $\mathrm{x}$ & & & \\
\hline M. furcata Ralfs & & $\mathrm{x}$ & $\mathrm{x}$ & & $\mathrm{X}$ & $\mathrm{X}$ & & & & & & \\
\hline M. laticeps Nordst. var. acuminata W. Krieg. & & $\mathrm{x}$ & & & & & & & & & & \\
\hline M. laticeps Nordst. var. laticeps & $\mathrm{x}$ & $\mathrm{x}$ & & & $\mathrm{x}$ & $\mathrm{x}$ & & & & $\mathrm{x}$ & $\mathrm{x}$ & \\
\hline M. mahabuleshwarensis Hobs. & & & $\mathrm{X}$ & & & & & & & & & \\
\hline M. mahabuleshwarensis Hobs. var. amazonensis Först. & & & & & & $\mathrm{x}$ & & & & & & \\
\hline M. mahabuleshwarensis Hobs. var. europaea (Turn.) W. Krieg. & & $\mathrm{x}$ & $\mathrm{x}$ & & & & & & & & & \\
\hline M.pinnatifida (Kütz.) ex Ralfs & & & $\mathrm{x}$ & & & & & & & & & \\
\hline M. radiosa Ralfs var. radiosa & & & & & & & & $\mathrm{x}$ & & & & \\
\hline M. radiosa Ralfs var. elegantior (G.S. West) Croasd. & & & & & & & & & & & $\mathrm{x}$ & \\
\hline M. rotata (Grev.) Ralfs ex Ralfs & & & & & & & & & $\mathrm{x}$ & & $\mathrm{x}$ & \\
\hline M. cf. torreyi Bail ex Ralfs var. nordstedtiana (Hieron.) Schmidle & & & & & & & & & $\mathrm{x}$ & & & \\
\hline M. truncata (Corda) Bréb. ex Ralfs var. truncata & & & $\mathrm{x}$ & & & & & & & & & \\
\hline M. truncata (Corda) Bréb. ex Ralfs var. pusilla G.S. West & $\mathrm{x}$ & $\mathrm{x}$ & $\mathrm{x}$ & $\mathrm{x}$ & & $\mathrm{x}$ & & & & & & \\
\hline Onychonema laeve Nordst. var. laeve & & & $\mathrm{X}$ & & & $\mathrm{x}$ & & & & & & \\
\hline O. laeve Nordst. var. latum West \& G.S.West & & $\mathrm{x}$ & & & $\mathrm{x}$ & & & & & & & \\
\hline O. laeve Nordst. var. rectangulare Grönbl. & & & $\mathrm{x}$ & & & $\mathrm{x}$ & & & & & & \\
\hline Pleurotaenium ehrenbergii (Bréb.) de Bary & & $\mathrm{x}$ & $\mathrm{x}$ & & $\mathrm{x}$ & & $\mathrm{X}$ & $\mathrm{x}$ & $\mathrm{x}$ & $\mathrm{x}$ & $\mathrm{x}$ & \\
\hline Sphaerozosma aubertianum W. West var. archeri (Gutw.) & & & & & & $\mathrm{X}$ & & & & & & \\
\hline
\end{tabular}


continuação

\begin{tabular}{|c|c|c|c|c|c|c|c|c|c|c|c|c|}
\hline \multirow{3}{*}{$\begin{array}{l}\text { Reservatórios } \\
\text { Períodos estacionais } \\
\text { Família/Táxons }\end{array}$} & \multicolumn{6}{|c|}{ Rosana } & \multicolumn{6}{|c|}{ Salto do Vau } \\
\hline & \multicolumn{3}{|c|}{ Verão } & \multicolumn{3}{|c|}{ Inverno } & \multicolumn{3}{|c|}{ Verão } & \multicolumn{3}{|c|}{ Inverno } \\
\hline & $\mathrm{F}$ & $\mathrm{T}$ & $\mathrm{L}$ & $\mathrm{F}$ & $\mathrm{T}$ & $\mathrm{L}$ & $\mathrm{F}$ & $\mathrm{T}$ & $\mathrm{L}$ & $\mathrm{F}$ & $\mathrm{T}$ & $\mathrm{L}$ \\
\hline Spondylosium moniliforme Lund. & & $\mathrm{x}$ & & & & $\mathrm{x}$ & & & & & & \\
\hline $\begin{array}{l}\text { S. panduriforme (Heimerl) Teil. var. limneticum (West \& } \\
\text { G.S. West) Först. }\end{array}$ & & $\mathrm{X}$ & & & $\mathrm{x}$ & & & & & & & \\
\hline S. papillosum West \& G.S. West & & & $\mathrm{x}$ & & & & & & & & & \\
\hline S. planum (Wolle) West \& G.S. West & & & $\mathrm{x}$ & & & & & & & & $\mathrm{x}$ & \\
\hline S. pulchrum Bail. & & & & & & $\mathrm{x}$ & & & & & & \\
\hline Spondylosium sp. & & & & & & $\mathrm{x}$ & & & & & & \\
\hline Staurastrum alternans (Bréb.) Ralfs & & & & & & & & $\mathrm{x}$ & & $\mathrm{x}$ & $\mathrm{x}$ & $\mathrm{x}$ \\
\hline S. ambiguum Turner & $\mathrm{x}$ & $\mathrm{x}$ & $\mathrm{x}$ & & $\mathrm{x}$ & $\mathrm{x}$ & & & & & & \\
\hline S. anatinum Cooke \& Wills & & & $\mathrm{x}$ & & & $\mathrm{x}$ & & & & & & \\
\hline S. bieneanum Rabenh. & $\mathrm{x}$ & $\mathrm{x}$ & $\mathrm{x}$ & & & & & & & & & \\
\hline S. boreale West \& G.S. West & & & $\mathrm{x}$ & & & $\mathrm{x}$ & & & & & & \\
\hline S. brasiliense Nordst. & & & & & & $\mathrm{x}$ & & & & & & \\
\hline S. cf. brebissonii Arch. & & & & & $\mathrm{x}$ & $\mathrm{x}$ & & $\mathrm{x}$ & & & & \\
\hline S. claviferum West \& G.S. West & $\mathrm{x}$ & $\mathrm{x}$ & $\mathrm{x}$ & & $\mathrm{x}$ & $\mathrm{x}$ & $\mathrm{x}$ & & & & $\mathrm{x}$ & \\
\hline $\begin{array}{l}\text { S. cyclacanthum West \& G.S. West var. americanum } \\
\text { Scott \& Grönbl. }\end{array}$ & $\mathrm{x}$ & & & & $\mathrm{x}$ & & & & & & & \\
\hline S. denticulatum (Näg.) Arch. & $\mathrm{x}$ & & & & & & & & & & & \\
\hline S. cf. dilatatum (Ehrenb.) Ralfs & $\mathrm{x}$ & $\mathrm{x}$ & $\mathrm{x}$ & $\mathrm{x}$ & $\mathrm{x}$ & $\mathrm{X}$ & & $\mathrm{x}$ & & & & \\
\hline S. cf. gracile Ralfs & & & & & & $\mathrm{x}$ & & & & & & \\
\hline S. hagmannii Grönbl. & $\mathrm{x}$ & & & & & & & & & & & \\
\hline S. hantzschii Reinsch & & & & & & & $\mathrm{x}$ & $\mathrm{x}$ & $\mathrm{x}$ & $\mathrm{x}$ & & \\
\hline S. leptacanthum Nordst. var. borgei Först. & & $\mathrm{x}$ & $\mathrm{x}$ & & & $\mathrm{x}$ & & & & & & \\
\hline S. leptocladum Nordst. var. leptocladum & $\mathrm{x}$ & & & & & & & & & & & \\
\hline S. leptocladum Nordst. var. cornutum Wille & & & & & $\mathrm{x}$ & & & & & & & \\
\hline S. margaritaceum (Ehrenb.) Ralfs & $\mathrm{X}$ & & & $\mathrm{x}$ & $\mathrm{x}$ & $\mathrm{X}$ & & & $\mathrm{x}$ & & $\mathrm{x}$ & \\
\hline S. muticum (Bréb.) Ralfs & & & & & & $\mathrm{x}$ & & & & & & \\
\hline S. nudibrachiatum Borge & & & $\mathrm{x}$ & & & $\mathrm{x}$ & & & & & & \\
\hline S. orbiculare (Ehrenb.) Ralfs var. orbiculare & & & & & & & & & & & $\mathrm{x}$ & \\
\hline S. orbiculare (Ehrenb.) Ralfs var. denticulatum Nordst. & & & $\mathrm{x}$ & & & $\mathrm{x}$ & & & & & & \\
\hline S. orbiculare (Ehrenb.) Ralfs var. depressum Roy \& Biss. & & $\mathrm{x}$ & $\mathrm{x}$ & & & $\mathrm{x}$ & & $\mathrm{x}$ & $\mathrm{x}$ & & & \\
\hline S. polymorphum Bréb. & & & & & $\mathrm{x}$ & $\mathrm{x}$ & & & & & & \\
\hline S. quadrangulare Bréb. ex Ralfs var. quadrangulare & $\mathrm{X}$ & $\mathrm{x}$ & $\mathrm{X}$ & $\mathrm{x}$ & & & & & & & & \\
\hline S. quadrangulare Bréb. ex Ralfs var. contectum (Turn.) & $\mathrm{x}$ & $\mathrm{x}$ & & & & & & & & & & \\
\hline S. quadricornnutum Roy \& Biss. & & $\mathrm{x}$ & $\mathrm{x}$ & & & & & & & & & \\
\hline S. cf. quadrinotatum Grönbl. & & & $\mathrm{x}$ & & & & & & & & & \\
\hline$S$. rotula Nordst. & & & $\mathrm{x}$ & & & & & & & & & \\
\hline S. sebaldi Reinsch var. ornatum Nordst. & $\mathrm{X}$ & & $\mathrm{X}$ & & $\mathrm{X}$ & $\mathrm{x}$ & & & & & & \\
\hline S. cf. setigerum Cleve & $\mathrm{x}$ & & $\mathrm{x}$ & & & & & & & & & \\
\hline S. cf. setigerum Cleve var. pectinatum West \& G.S. West & $\mathrm{X}$ & & $\mathrm{x}$ & & $\mathrm{X}$ & $\mathrm{x}$ & & $\mathrm{x}$ & $\mathrm{x}$ & & & \\
\hline S. tetracerum (Kütz.) Ralfs & & & $\mathrm{x}$ & & & $\mathrm{x}$ & & & & & & \\
\hline S. trifidum Nordst. var. inflexum West \& G.S. West & $\mathrm{x}$ & & $\mathrm{x}$ & & & & & $\mathrm{x}$ & & & & \\
\hline Staurodesmus cf. controversus (W. West) Teil. & & & $\mathrm{x}$ & & & & & & & & & \\
\hline S. convergens (Ehrenb. ex Ralfs) S. Lill. & & & $\mathrm{x}$ & & & & & & & & & \\
\hline S. corniculatus (Lund.) Teil. & & & $\mathrm{x}$ & & & & & & & & & \\
\hline S. corniculatus (Lund.) Teil. var. spinigerum W. West & & $\mathrm{X}$ & $\mathrm{x}$ & & & & & & & & & \\
\hline S. cf. cornutus (Wolle) Teil. & & & $\mathrm{X}$ & & & & & & & & & \\
\hline S. cuspidatus (Bréb. ex Ralfs) Teil. var. cuspidatus & & & $\mathrm{x}$ & & & $\mathrm{x}$ & & & & & & \\
\hline S. cuspidatus (Bréb. ex Ralfs) Teil. var. maximus W. West & & & & & & $\mathrm{x}$ & & & & & & \\
\hline
\end{tabular}


continuação

\begin{tabular}{|c|c|c|c|c|c|c|c|c|c|c|c|c|}
\hline \multirow{3}{*}{$\begin{array}{l}\text { Reservatórios } \\
\text { Períodos estacionais } \\
\text { Família/Táxons }\end{array}$} & \multicolumn{6}{|c|}{ Rosana } & \multicolumn{6}{|c|}{ Salto do Vau } \\
\hline & \multicolumn{3}{|c|}{ Verão } & \multicolumn{3}{|c|}{ Inverno } & \multicolumn{3}{|c|}{ Verão } & \multicolumn{3}{|c|}{ Inverno } \\
\hline & $\mathrm{F}$ & $\mathrm{T}$ & $\mathrm{L}$ & $\mathrm{F}$ & $\mathrm{T}$ & $\mathrm{L}$ & $\mathrm{F}$ & $\mathrm{T}$ & $\mathrm{L}$ & $\mathrm{F}$ & $\mathrm{T}$ & $\mathrm{L}$ \\
\hline S. dejectus (Bréb.) Teil. var. dejectus & & & $\mathrm{x}$ & & & & & & & & & \\
\hline S. dejectus (Bréb.) Teil. var. apiculatus (Bréb.) Teil. & & & & & & & & & $\mathrm{x}$ & & & \\
\hline S. dickiei (Ralfs) S. Lill. var. dickiei & & $\mathrm{x}$ & $\mathrm{x}$ & & & $\mathrm{x}$ & & $\mathrm{x}$ & & $\mathrm{x}$ & $\mathrm{x}$ & $\mathrm{x}$ \\
\hline S. dickiei (Ralfs) S. Lill. var. maximus (West \& G.S. West) Thom. & & $\mathrm{X}$ & & & & & & & & & & \\
\hline S. cf. extensus (Borge) Teil. & & & $\mathrm{x}$ & & & $\mathrm{x}$ & & & & & & \\
\hline S. glaber (Ehrenb. ex Ralfs) S. Lill. & & & $\mathrm{x}$ & & & & & & & & & \\
\hline $\begin{array}{l}\text { S. lanceolatus (Arch.) Croasd. var. rotundatus (Messik.) } \\
\text { Croasd. }\end{array}$ & & & & & & & & $\mathrm{x}$ & & & & \\
\hline S. lobatus (Börges.) Bourr. & & & $\mathrm{x}$ & & & $\mathrm{X}$ & & & & & & \\
\hline S. mamillatus (Nordst.) Teil. & & & $\mathrm{x}$ & & & & & & & & & \\
\hline $\begin{array}{l}\text { S. mucronatus (Borge) Teil. var. subtriangularis (W. West) } \\
\text { Croasd. }\end{array}$ & & & $\mathrm{x}$ & & & & & & & & & \\
\hline S. triangulares (Lagerh.) Teil. & & & $\mathrm{x}$ & & & & & & & & & \\
\hline Staurodesmus sp. & & & & & & $\mathrm{x}$ & & & & & & \\
\hline Desmidiaceae não identificada & & & & & & $\mathrm{X}$ & & & $\mathrm{x}$ & & $\mathrm{x}$ & \\
\hline
\end{tabular}

$*$ espécies cosmopolitas, $* *=$ forma menor

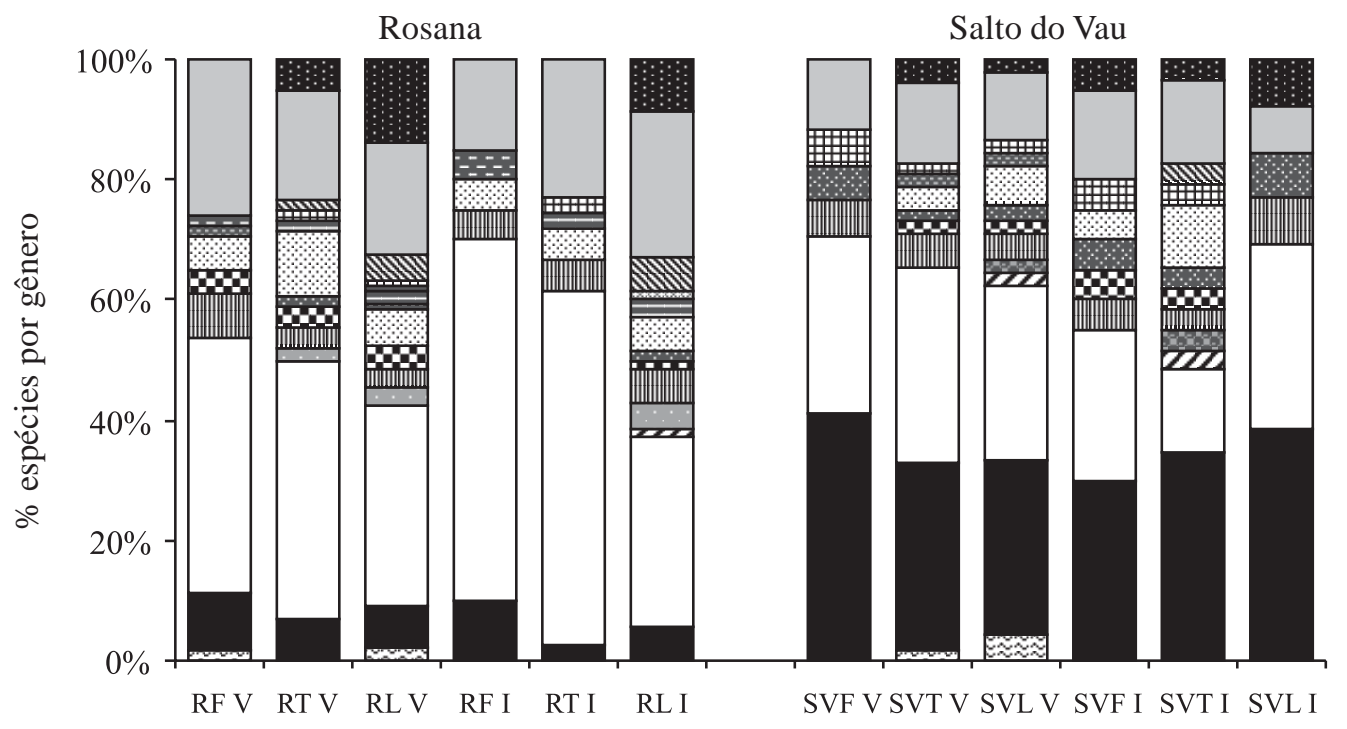

\begin{tabular}{|c|c|c|c|}
\hline 웅 Actinotaenium & Closterium & $\square$ Cosmarium & Ⓒosmocladium \\
\hline 圆Cilyndrocystis & $\square$ Desmidium & 血 Euastrum & $\boxminus$ Gonatozygon \\
\hline 圆Hyalotheca & 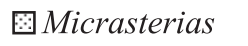 & 圖 Netrium & 目Onychonema \\
\hline $\begin{array}{l}\text { 国enium } \\
\square \text { Staurastrum }\end{array}$ & $\begin{array}{l}\text { 团Pleurotaenium } \\
\text { Staurodesmus }\end{array}$ & 图 Sphaerozosma & 图 Spondylosium \\
\hline
\end{tabular}

Figura 3. Porcentagem de espécies de desmídias por gênero encontrada nas diferentes regiões: fluvial (F), transição (T), lacustre (L), no verão (V) e inverno (I), em ambos os reservatórios (R = Rosana, SV = Salto do Vau) no ano de 2002.

Figure 3. Specific percentage of desmids for genus found in the various regions: fluvial (F), transition (T) and lacustrine (L), in the summer (V) and winter (I), in both the reservoirs (R = Rosana, SV = Salto do Vau) in the year of 2002. 


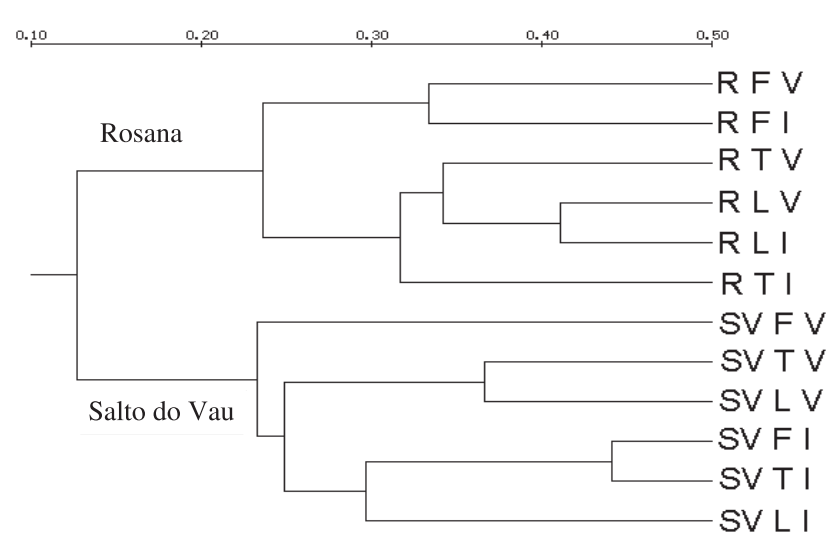

Figura 4. Dendrograma obtido por UPGMA, resultante da análise de agrupamento baseado no índice de similaridade de Jaccard, entre as espécies de desmídias. Teste de Mantel com $r=0,87$. Regiões: fluvial (F), transição (T), lacustre (L); período estacional: verão (V) e inverno (I).

Figure 4. Dendrogram obtained for UPGMA, resultant of the analysis of grouping based on Jaccard's similarity index, between the species of desmids. Mantel test with $r=0.87$. Regions: Fluvial (F), transition (T) and lacustrine (L); seasonal period: summer (V) and winter (I).

tanto no verão quanto no inverno, constatou-se maior riqueza de macrófitas e de desmídias perifíticas. Essa relação não ficou evidenciada na região fluvial deste reservatório, onde foi registrado um número elevado de macrófitas, mas um baixo número de desmídias (figura 5).

\section{Discussão}

A composição de desmídias perifíticas foi dissimilar entre os reservatórios e suas regiões ao longo da distribuição longitudinal (rio-barragem). A diferença entre períodos estacionais, alterando a composição de desmídias, ficou evidenciada no Reservatório de Salto do Vau, onde existe uma diferença maior de temperatura entre verão e inverno. A localização de reservatórios em bacias hidrográficas distintas, bem como a presença ou ausência de macrófitas aquáticas, afetaram o conjunto de desmídias perifíticas.

A maior riqueza de táxons de desmídias em Rosana, quando comparado a Salto do Vau, pode estar relacionada às diferenças de localização dos reservatórios, à riqueza de macrófitas e aos usos múltiplos da bacia de drenagem de cada reservatório. Segundo Tundisi (1999) e Tundisi et al. (1999), a localização e o tamanho do reservatório na bacia de drenagem, o tempo de permanência da água e os fatores

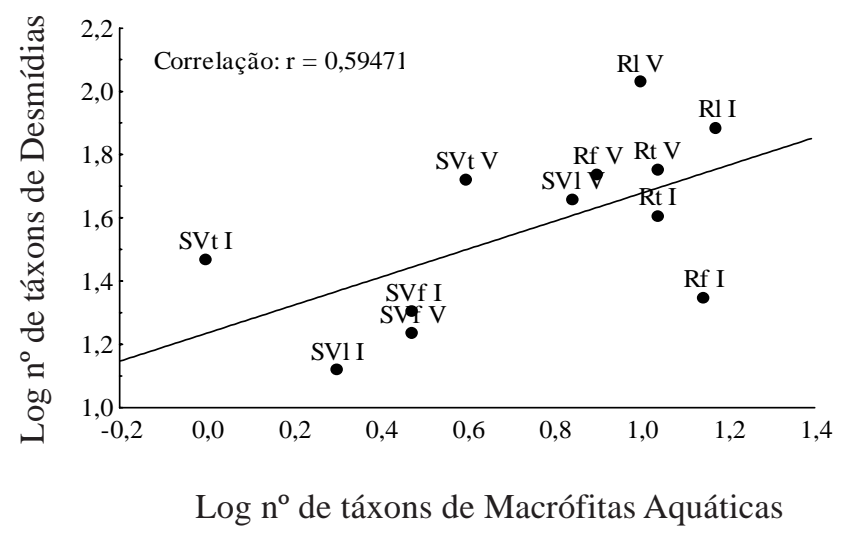

Figura 5. Correlação de Pearson entre a riqueza de táxons de desmídias e de macrófitas aquáticas, referente a dois períodos estacionais (verão $=\mathrm{V}$, inverno $=\mathrm{I}$ ), nos reservatórios de Rosana (R) e Salto do Vau (SV) e nas diferentes regiões: fluvial (f), transição (t) e lacustre (1).

Figure 5. Pearson correlation analysis between the richness of desmids and aquatic macrophytes taxa, referring the two periods (summer $=\mathrm{V}$, winter $=\mathrm{I}$ ), in Rosana $(\mathrm{R})$ and Salto do Vau (SV) reservoirs and in the various regions: fluvial (f), transition (t) and lacustrine (l).

climáticos são características que afetam a diversidade biológica. Thomaz \& Bini (2003) afirmam que a profundidade reduzida, a elevada transparência da água, a baixa declividade e o maior desenvolvimento das margens contribuem para o estabelecimento de plantas aquáticas, características essas observadas em Rosana e, conseqüentemente, constituem excelentes hospedeiros para as algas epifíticas.

A maior riqueza de desmídias observada na região lêntica em Rosana se deve, possivelmente, aos valores mais elevados de temperatura, $\mathrm{pH}$, condutividade e maior riqueza de macrófitas, principalmente no verão. De acordo com Coesel (1982), a composição e a abundância de desmídias estão mais correlacionadas com $\mathrm{pH}$, condutividade e presença de macrófitas. A presença de macrófitas estimula o desenvolvimento de algas perifíticas, devido ao fornecimento de substrato para fixação e pela grande quantidade de material nutritivo proveniente do seu próprio processo de envelhecimento e morte (Chamixaes 1991, Schwarzbold 1992, Tell et al. 1994, Taniguchi 1998). Ainda, Rodrigues \& Bicudo (2001) relacionaram a grande riqueza de desmídias perífiticas à presença de estandes de macrófitas aquáticas.

De acordo com Toja \& Casco (1991), a flutuação do nível da água, o impedimento da instalação de macrófitas, a baixa transparência da água, a instabilidade 
de substratos e o grau de inclinação do rio restringem o desenvolvimento do perifíton em reservatórios. Em estudos de variação sazonal de regiões temperadas, Canter \& Lund (1966), Sommer (1981) e Coesel \& Wardenaar (1990) mostraram que o pico de desenvolvimento das desmídias geralmente ocorre no final do verão e início do outono, podendo apresentar diferenças bastante acentuadas entre as espécies. Esse fato ocorre, possivelmente, devido à variação da temperatura entre $25-30{ }^{\circ} \mathrm{C}$ que, conforme Coesel \& Wardenaar (1990), é considerada ótima para o crescimento desse grupo. Segundo Coesel (1996), temperaturas mais elevadas explica o fato das desmídias terem se originado em regiões tropicais, já que nessas regiões a flora de desmídias é mais diversificada, tanto em espécies como em gêneros. Assim, para o Reservatório de Salto do Vau, sistema mais encaixado, com as margens sombreadas pela vegetação terrestre muito abundante, com uma menor riqueza de macrófitas, baixa transparência da água, aliadas aos menores valores de temperatura e condutividade, podem estar influenciando na menor riqueza de desmídias.

A predominância de espécies de Cosmarium no Reservatório de Rosana pode ser devido ao hábito cosmopolita do gênero, já que muitos de seus táxons ocorrem em ambientes com águas mais claras e abundância de plantas submersas (Coesel 1982, Förster 1982, Bicudo \& Ungaretti 1986, Bourrely \& Couté 1991, Franceschini 1992, Tell et al. 1994) e em ambientes com situações eutróficas (Prescott et al. 1981). O gênero Closterium, com maior riqueza em Salto do Vau, apresenta muitas espécies resistentes e bem adaptadas às condições de baixa intensidade luminosa e maior quantidade de matéria orgânica (Brook \& Williamson 1988). A predominância de Closterium pode estar relacionada com a capacidade de movimentação, que ocorre através de fototaxia positiva e, também, pela produção de bainhas mucilaginosas (Evans 1958).

No diagrama da análise de agrupamento, a separação dos períodos estacionais no Reservatório de Salto do Vau se deve, provavelmente, à temperatura, a qual foi mais elevada no verão e mais baixa no inverno. A maior similaridade entre as regiões deve estar relacionada ao ciclo hidrológico da bacia do Rio Iguaçu, que apresenta maior índice pluviométrico no verão e menor no inverno. Assim, o reservatório deve aumentar em extensão a região lótica no inverno, levando conseqüentemente a uma maior similaridade florística entre as regiões fluvial e transição. Já no verão, devido às chuvas, ocorre maior retenção de água no reservatório, com aumento da zona lacustre. Dessa forma, a maior similaridade entre as desmídias perifíticas ocorre entre as regiões de transição e lêntica.

De acordo com Nogueira et al. (2001), os reservatórios da Bacia do Paranapanema apresentam um padrão de variação definido pela alternância entre o período chuvoso no verão e seco no inverno. Acreditase que esse fator foi fundamental para a separação da região de transição das demais regiões do Reservatório de Rosana. Dessa forma, no verão, com a elevação do nível de água, a região de transição adquire condições mais lênticas, fazendo com que a comunidade de desmídias seja mais similar às da região lêntica. A velocidade de corrente representa uma das principais funções de força atuantes em reservatórios, exercendo uma grande influência na dinâmica da região fluvial. Assim, a velocidade de corrente pode dificultar a permanência das algas frouxamente aderidas ao perifíton, como é o caso das desmídias. Isso, provavelmente, justifica a maior similaridade entre as desmídias na região fluvial de ambos os períodos.

Agradecimentos - Este trabalho está inserido no Programa de Pesquisas Pronex: Produtividade em Reservatório: relações com o estado trófico e predação; e CT-Hidro: Identificação de indicadores biológicos de eutrofização e poluição de reservatórios da bacia do rio Paraná, executados pelo Núcleo de Pesquisas em Limnologia, Ictiologia e Aqüicultura Nupélia, da Universidade Estadual de Maringá. Agradecemos de forma especial ao professor Dr. Carlos Eduardo de Mattos Bicudo pelo auxílio na identificação e discussão taxonômica, aos biólogos e técnicos do Nupélia, pelo suporte técnicocientífico, ao Jaime Luiz Lopes Pereira pela confecção do mapa, a Copel (Companhia Paranaense de Energia) pelo suporte logístico e ao CNPq pela concessão da bolsa de mestrado.

\section{Referências bibliográficas}

BICUDO, C.E.M. \& UNGARETTI, I. 1986. Desmídias (Zygnemaphyceae) da lagoa-represa de Águas Belas, Rio Grande do Sul, Brasil. Revista Brasileira de Biologia 46:285-307.

BICUDO, C.E.M., BICUDO, D.C., CASTRO,A.A.J. \& PICELLIVICENTIM, M.M. 1992. Fitoplâncton do trecho a represar do rio Paranapanema (Usina Hidrelétrica de Rosana), Estado de São Paulo, Brasil. Revista Brasileira de Biologia 52:293-310.

BICUDO, D.C., BICUDO, C.E.M., CASTRO, A.A.J. \& PICELLI-VICENTIM, M.M. 1993. Diatomáceas (Bacillariophyceae) do trecho a represar do rio Paranapanema (Usina Hidrelétrica de Rosana), Estado de São Paulo, Brasil. Hoehnea 20:47-68.

BOURRELLY, P. \& COUTÉ, A. 1991. Desmidiées de Madagascar (Chlorophyta, Zygophyceae). Bibliotheca phycologica, v.86. J. Cramer, Berlin. 
BROOK, J.A. 1981. The Biology of desmids. Blackwell Scientific Publications, Oxford.

BROOK, J.A. \& WILLIAMSON, D.B. 1988. The Survival of Desmids on the Drying Mud of a Small Lake. In Algae and the Aquatic Environment. (F.E. Round, ed.). Biopress, Bristol, p.185-196.

CANTER, H.M. \& LUND, J.W.G. 1966. The periodicity of planktonic desmids in Windermere, England. Verhandlungen der Internationale Vereinigung für Limnologie 16:163-172.

CHAMIXAES, C.B.C.B. 1991. Variação temporal e espacial da biomassa, composição de espécies e produtividade das algas perifíticas relacionadas com as condições ambientais de pequenos rios da bacia hidrográfica do Ribeirão do Lobo (Itirapina - SP). Tese de doutorado, Universidade Federal de São Carlos, São Carlos.

COESEL, P.F.M. 1982. Structural characteristics and adaptations of desmid communities. Journal of Ecology 70:163-177.

COESEL, P.F.M. 1996. Biogeography of desmids. Hydrobiologia 336:41-53.

COESEL, P.F.M. \& WARDENAAR, K. 1990. Growth responses of planktonic desmid species in a temperature - light gradient. Freshwater Biology 23:551-560.

CROASDALE, H. \& FLINT, E.A. 1986. Flora of New Zealand: Freshwater algae, Chlorophyta, Desmids. Government Printer, Wellington.

CROASDALE, H. \& FLINT, E.A. 1988. Flora of New Zealand: Freshwater algae, Chlorophyta, Desmids. Government Printer, Wellington.

DILLARD, G.E. 1990. Freshwater Algae of the Southeastern United States. Part 3. Chrolophyceae: Zygnematales: Mesotaeniaceae and Desmidiaceae (Section 1). Bibliotheca phycologica, v.85. J. Cramer, Berlin.

DILLARD, G.E. 1991. Freshwater Algae of the Southeastern United States. Part 4. Chrolophyceae: Zygnematales: Desmidiaceae (Section 2). Bibliotheca phycologica, v.89. J. Cramer, Berlin.

EVANS, J.H. 1958. The survival of freshwater algae during dry periods. Part I An investigation of the algae of five small ponds. Journal of Ecology 46:149-167.

FRANCESCHINI, I.M. 1992. Algues d'eau douce de Porto Alegre, Brésil (les Diatomophycées exclues). Bibliotheca phycologica, v.92. J. Cramer, Berlin.

FÖRSTER, K. 1982. Conjugatophyceae: Zygnematales und Desmidiales (excl. Zygnemataceae). In Das Phytoplankton des Süsswassers: Systematik und Biologie (G. Huber-Pestalozzi, ed.). Schweizerbart'sche Verlagsbuchhandlung, Stuttgart.

GOLDSBOROUGH, G. \& ROBINSON, G.G.C. 1996. Pattern in Wetlands. In Algal Ecology: Freshwater Benthic Ecosystems (R.J. Stevenson, M.L. Bothwell \& R.L. Lowe, eds.). Academic Press, San Diego. 753p.

HUSZAR, V.L.M. 1994. Fitoplâncton de um lago amazônico impactado por rejeito de bauxita (lago Batata, Pará, Brasil): estrutura da comunidade, flutuações espaciais e temporais. Tese de doutorado, Universidade Federal de São Carlos, São Carlos.
NOGUEIRA, M.G., VIANA, N.C., JORCIN, A. \& BRITTO, Y.T. 2001. Limnologia comparada de 8 reservatórios em cascata no rio Paranapanema (SP-PR), Brasil. In Seminário Internacional Gestión Ambiental e Hidroelectricidad. Disponível em CD-Rom. Salto, Argentina - Uruguay, CACIER-CTMSG. p.1-20.

PRESCOTT, G.W., CROASDALE, H.T., VINYARD, W.C. \& BICUDO, C.E.M. 1981. A Synopsis of North American Desmids; Part II. Desmidiaceae: Placodermae. Section 3. In Desmidiales. (G.W. Prescott, ed.). University Nebraska Press, Lincoln, 720p.

PRESCOTT, GW., BICUDO, C.E.M. \& VINYARD, W.C. 1982.A Synopsis of North American Desmids. Part II. Desmidiaceae: Placodermae. Section 4. In Desmidiales. (G.W. Prescott, ed.). University Nebraska Press, Lincoln, 698p.

RODRIGUES, L. \& BICUDO, D.C. 2001. Similarity among periphyton algal communities in a lentic-lotic gradient of the upper Paraná river floodplain, Brazil. Revista Brasileira de Botânica 24:235-248.

RODRIGUES, L., BICUDO, D.C. \& MOSCHINI-CARLOS, V. 2003. O papel do perifíton em áreas alagáveis e nos diagnósticos ambientais. In Ecologia e Manejo de Macrófitas Aquáticas (S.M. Thomaz \& L.M. Bini, eds.). Eduem, Maringá, p.211-229.

ROHLF, F. J. 1989. NTSYS-PC. Numerical taxonomy and multivariate analysis system, version 1,50. Exeter Publ. Ltda.

SCHWARZBOLD, A. 1992. Efeitos do regime de inundação do rio Mogi-guaçu (SP) sobre a estrutura, diversidade, produção e estoques do perifíton da Lagoa do Infernão. Tese de doutorado, Universidade Federal de São Carlos, São Carlos.

SLÁDECKOVÁ, A. 1962. Limnological investigation methods for the periphyton ("Aufwuchs") community. Botanical Review 28:286-350.

SOMMER, U. 1981. The role of r- and k-selection in the sucession of phytoplankton in Lake Constance. Acta Oecologica 2:327-342.

STATSOFT. 1996. Statistica. Tulsa 3. Systat Inc., Evanston.

TANIGUCHI, G.M. 1998. Variação espacial e temporal de características limnológicas abióticas e de comunidades de algas planctônicas e perifíticas no gradiente litorâneolimnético de uma lagoa marginal do rio Mogi-Guaçu. Tese de mestrado, Universidade Federal de São Carlos, São Carlos.

TANIGUCHI, G.M., BICUDO, D.C. \& SENNA, P.A.C. 2000. Intercâmbio populacional de desmídias planctônicas e perifíticas na lagoa do Diogo, Planície de Inundação do rio Mogi-guaçu. In Estação Ecológica de Jataí: estudos integrados em ecossistemas. (J.E. Santos \& J.S.R. Pires, eds.). EDUSCar, São Carlos, v.2, p.431-444.

TELL, G., IZAGUIRRE, I. \& O`FARRELL, I. 1994. Ecological and Taxonomical Remarks on the Desmid Flora of the Lower Uruguay River Basin (Argentina). Bibliotheca phycologica, v.96. J. Cramer, Berlin. 
THOMAZ, S.M. \& BINI, L.M. 2003. Ecologia e Manejo de Macrófitas Aquáticas. Eduem, Maringá.

THOMAZ, S.M., BINI, L.M. \& ALBERT, S.M. 1997. Limnologia do reservatório de Segredo: padrões de variação espacial e temporal. In Reservatório de Segredo: bases ecológicas para o manejo (A.A. Agostinho \& L.C. Gomes, eds.). Eduem, Maringá, p. 19-37.

THORNTON, K.W. 1990. Perspectives on Reservoir Limnnology. In Reservoir Limnnology: Ecological Perspectives (K.W. Thornton, B.L. Kimmel \& F.E. Payne, eds.). John Wiley \& Sons, New York, p.1-15.

TOJA, J. \& CASCO, M.A. 1991. Contribution of phytoplankton and periphyton to the production in a reservoir of S. W. Spain. Oecologia Aquatica 10:61-76.

TUNDISI, J.G. 1999. Reservatórios como sistemas complexos: Teoria, aplicações e perspectivas para usos múltiplos. In Ecologia de reservatórios: estrutura, funções e aspectos sociais (R. Henry, ed.). Fundbio / Fapesp, Botucatu / São Paulo, p.19-38.
TUNDISI, J.G., MATSUMURA-TUNDISI, T. \& ROCHA, O. 1999. Theoretical basis for reservoir management. In Theoretical reservoir ecology and its applications. (J.G. Tundisi \& M. Straskraba, eds.). International Institute of Ecology, São Carlos and Backhuys Publishers, AH Leiden, p.505-528.

ZANATA, L.H. \& ESPÍNDOLA, E.L.G. 2002. Longitudinal processes in Salto Grande reservoir (Americana, SP, Brazil) and its influence in the formation of compartment system. Brazilian Journal of Biology 62:347-361.

WEITZEL, R.L. 1979. Periphyton measurements and application. In Methods and measurements of periphyton communities: a review. (R.L. Weitzel, ed.). American Society for Testing and Materials, Philadelphia, p.3-33.

WETZEL, R.G. 1983. Recommendations for future research on periphyton. In Periphyton of freshwater ecosystems (R.G. Wetzel, ed.). Developments in Hidrobiology, 17. Dr. W. Junk, The Hague, p.339-346. 